\title{
Análise do comportamento de membranas cerâmicas de $\alpha$-alumina/Pd para separação de gases a temperatura ambiente e baixas pressões
}

\section{(Membrane behavior analysis of $\alpha$-alumina/Pd for gas separation at ambient temperature and low pressures)}

\author{
R. Bertotto ${ }^{1}$, J. Duarte ${ }^{1}$, V. dos Santos ${ }^{1 *}$, M.Zeni ${ }^{1}$, C.P. Bergmann ${ }^{2}$ \\ ${ }^{1}$ Universidade de Caxias do Sul, R. Francisco Getúlio Vargas 1130, Caxias do Sul, RS, Brasil \\ ${ }^{2}$ Universidade Federal do Rio Grande do Sul, Escola de Engenharia, Porto Alegre, Brasil
}

\begin{abstract}
Resumo
Membranas de compósitos $\mathrm{Pd} / \alpha-\mathrm{Al}_{2} \mathrm{O}_{3}$ com uma e duas camadas de Pd foram preparadas pelo processo electroless plating sobre tubos cerâmicos porosos. A análise de espectroscopia por dispersão de energia confirmou a composição química do suporte cerâmico (Al e O) e da membrana com Pd (Al, O e Pd), bem como a espessura da camada de Pd de 0,8 $\mu$ m (uma camada) e 1,3 $\mu \mathrm{m}$ (duas camadas). As imagens de microscopia eletrônica de varredura mostraram que a superfície da membrana foi mais uniforme com uma camada do que duas camadas de $\mathrm{Pd}$. O difratograma de raios X registrou picos referentes à fase $\alpha$-alumina. A porosimetria por intrusão de mercúrio apresentou diâmetro de poro em torno de $5 \mathrm{~nm}$ e volume máximo de $\mathrm{Hg}$ intrudado de $0,0081 \mathrm{~cm}^{3} . \mathrm{g}^{-1}$. Os testes de permeabilidade aos gases mostraram que as membranas possuem seletividade $\alpha$ de 3,79 para o par $\mathrm{H}_{2} / \mathrm{CO}_{2}$ com um camada de Pd e de $\alpha=3,43$ para o par $\mathrm{H}_{2} / \mathrm{CH}_{4}$ com duas camadas de $\mathrm{Pd}$.

Palavras-chave: membranas cerâmicas, $\mathrm{Pd} / \alpha-\mathrm{Al}_{2} \mathrm{O}_{3}$, electroless plating, separação de gás.
\end{abstract}

Abstract

Composite membranes $\mathrm{Pd} / \mathrm{\alpha}-\mathrm{Al}_{2} \mathrm{O}_{3}$ with one and two Pd layers were prepared by electroless plating process on porous ceramic tubes. The analysis by energy dispersive spectroscopy confirmed the chemical composition of the ceramic support (Al and $O$ ) and of the membrane with $\mathrm{Pd}(\mathrm{Al}, \mathrm{O}$ and $\mathrm{Pd}$ ), as well the thickness of Pd layer of $0.8 \mu \mathrm{m}$ (one layer) and $1.3 \mu \mathrm{m}$ (two layers). Scanning electron microscopy images showed that the surface membrane was more uniform with one layer than two Pd layers. The X-ray diffraction pattern recorded peaks assigned to $\alpha$-alumina phase. Mercury intrusion porosimetry showed a pore diameter around $5 \mathrm{~nm}$ and maximum intruded $\mathrm{Hg}$ volume of $0.0081 \mathrm{~cm}^{3} . \mathrm{g}^{-1}$. Gas permeability tests showed that the membranes had selectivity $\alpha$ of 3.79 for $\mathrm{H}_{2} / \mathrm{CO}_{2}$ pair with one Pd layer and $\alpha=3.43$ for $\mathrm{H}_{2} / \mathrm{CH}_{4}$ pair with two Pd layers.

Keywords: $\mathrm{Pd} / \alpha-\mathrm{Al}_{2} \mathrm{O}_{3}$, ceramic membranes, electroless plating, gas separation.

\section{INTRODUÇÃO}

A emissão de poluentes na atmosfera é um problema global grave sendo que grande parte destas emissões ocorrem devido à queima de combustíveis fósseis. Para amenizar este problema, pesquisadores estão à procura de compostos que possibilitem uma combustão limpa e sem poluentes [1]. Dentre as possibilidades destaca-se o hidrogênio $\left(\mathrm{H}_{2}\right)$, que é 50\% mais eficiente em relação aos demais combustíveis, como a gasolina e o etanol, e produz como subproduto apenas a água [2]. A maior parte da produção de hidrogênio em escala industrial é feita pelo processo de reforma a vapor de metano $\left(\mathrm{CH}_{4}\right)$. Esse processo é muito endotérmico e, por isso, necessita de grandes quantidades de energia para produzir altas conversões, ocasionando maior custo no produto final [2]. Os processos de separação por membranas (PSM) são vistos como uma tecnologia promissora para diminuir estes custos devido ao fácil escalonamento de

*vsantos2@ucs.br módulos e a baixa energia necessária para operação do sistema. Nestes processos, a seletividade e a permeabilidade da membrana são características fundamentais para aumentar a eficiência da separação [3]. O uso do paládio (Pd) e suas ligas em membranas têm sido amplamente investigadas para a separação de hidrogênio devido à permisseletividade e estabilidade térmica e química. O Pd se sobressai como o metal com maior afinidade ao hidrogênio devido à alta reatividade em relação aos demais metais [4]. O Pd possui a habilidade de dissociar o hidrogênio na forma molecular através de sua estrutura cristalina. Em sua forma metálica, absorve hidrogênio gasoso em quantidade de até 600 vezes o seu volume mantendo as propriedades físicas e a integridade estrutural tornando-o eficiente na separação de hidrogênio de mistura de gases [5].

O processo de transporte do hidrogênio segue o mecanismo solução-difusão [2], o qual ocorre pela dissociação do gás no material da membrana formando uma solução homogênea sem depender da estrutura do mesmo e se difundindo através da membrana [6]. A permeação do hidrogênio através do 
Pd é um processo complexo e ocorre do lado de maior para a de menor pressão parcial, consistindo da adsorção e dissociação das moléculas de hidrogênio, seguida da difusão do átomo de hidrogênio através da rede cristalina do metal e recombinação dos átomos de hidrogênio no lado de menor pressão e dessorção do hidrogênio [7]. Dependendo da combinação das propriedades da membrana de Pd e condições operacionais, diferentes etapas limitantes do processo podem prevalecer. Os processos mais comuns são a difusão no interior do metal e a reação de superfície. Em condições operacionais de elevadas temperaturas prevalece a difusão no interior do metal, mas em temperaturas menores a reação que ocorre na superfície é a etapa determinante [8].

A força motriz para a separação de gases é o gradiente de potencial químico. Neste caso, a separação dos gases ocorre pela solubilidade e difusividade dos materiais pela membrana [9]. Fick, em 1855, propôs uma equação de difusão em estado estacionário considerando a força motriz como o gradiente de concentração, conforme:

$$
\mathrm{J}_{\mathrm{H}}=-\mathrm{D} \cdot \frac{\mathrm{dC}_{\mathrm{H}}}{\mathrm{d}_{\mathrm{Z}}}
$$

sendo $\mathrm{J}_{\mathrm{H}}$ o fluxo de hidrogênio através da membrana, D o coeficiente de difusão, $C_{H}$ é a concentração de hidrogênio e Z é a espessura da camada de Pd da membrana onde a difusão é considerada. O sinal negativo da Eq. A indica que o fluxo se encontra na direção contrária ao gradiente de concentração [10]. O mecanismo de difusão-solução define a permeabilidade (P) como o produto da difusividade (D) e da solubilidade $(\mathrm{S})$, de acordo com:

$$
\mathrm{P}=\mathrm{D} \cdot \mathrm{S}
$$

Os processos experimentais conhecidos até hoje determinam a permeabilidade, a solubilidade $\mathrm{e}$ a difusividade assumindo que o transporte de hidrogênio é governado pela difusão do $\mathrm{H}$ através do metal e que são fenômenos termicamente ativos. A permeabilidade pode ser calculada por:

$$
\mathrm{P}=\frac{\mathrm{V} \cdot \mathrm{e}}{\mathrm{t} \cdot \mathrm{A} \cdot \Delta \mathrm{P}}
$$

sendo $\mathrm{P}$ o coeficiente de permeabilidade (barrer), $\mathrm{V}$ o volume $(\mathrm{mL})$, e a espessura da camada de $\mathrm{Pd}(\mathrm{cm}), \mathrm{t}$ o tempo (s), $\mathrm{A}$ a área da membrana $\left(\mathrm{cm}^{2}\right)$ e $\Delta \mathrm{P}$ a diferença de pressão $(\mathrm{cmHg})$. A seletividade da membrana geralmente é expressa por um dos seguintes parâmetros: retenção (R) ou fator de separação $(\alpha)$. A retenção é calculada conforme Eq. D e o fator de separação calculado conforme Eq. E [11]:

$$
\mathrm{R}=1-\frac{\mathrm{Cp}}{\mathrm{Cf}}
$$

sendo $C_{p}$ a concentração de soluto no permeado e $C_{f}$ concentração na alimentação. Quando $\mathrm{R}=1$ há total retenção do soluto, sendo uma membrana ideal, logo quando $\mathrm{R}=0$ soluto e solvente permeiam livremente através da membrana [11];

$$
\alpha_{A / B}=\frac{y_{A} / y_{B}}{x_{A} / x_{B}}
$$

sendo $\mathrm{y}_{\mathrm{A}}$ e $\mathrm{y}_{\mathrm{B}}$ as concentrações molares de $\mathrm{A}$ e $\mathrm{B}$ no permeado, e $\mathrm{x}_{\mathrm{A}}$ e $\mathrm{x}_{\mathrm{B}}$ são as concentrações molares de $\mathrm{A}$ e $\mathrm{B}$ na alimentação.

O desenvolvimento de membranas permisseletivas com Pd e suas ligas está focado em obter resistência mecânica adequada e redução da espessura da camada seletiva. O desenvolvimento de uma membrana com Pd depende de uma fina e permisseletiva camada de $\mathrm{Pd}$, a qual é determinada pela seleção de um substrato poroso com alta área superficial e baixa resistência à permeação [12]. Em concordância com a literatura, a temperatura e a pressão utilizadas nos processos PSM apresentam significante influência nos resultados obtidos. Tanaka et al. [13] testaram o fluxo permeado em membranas de paládio com suporte de $\alpha$-alumina a 300 ${ }^{\circ} \mathrm{C}$ e pressão de entrada entre 100 a $300 \mathrm{kPa}$ e obtiveram resultados de $0,6 \mathrm{~mol} \cdot \mathrm{m}^{-2} \cdot \mathrm{s}^{-1}$. Yun et al. [14] obtiveram fluxo permeado de 0,05 a $0,45 \mathrm{~mol} \cdot \mathrm{m}^{-2} \cdot \mathrm{s}^{-1}$ para condições de 0,3 a 1,2 bar a $460{ }^{\circ} \mathrm{C}$. A permeabilidade de hidrogênio no paládio aumenta com a temperatura, visto que a energia de ativação endotérmica para a difusão domina a adsorção do hidrogênio no paládio [15]. No entanto, de acordo com [16], o aumento da temperatura resulta na diminuição do fluxo de hidrogênio com o passar do tempo.

A possibilidade de separação do hidrogênio devido à sua permeação através da membrana muda as limitações termodinâmicas existentes, pois desloca o equilíbrio da reação no sentido da formação dos produtos, permitindo que a mesma se realize em temperaturas mais moderadas e com conversões maiores [17]. Ainda, a separação do $\mathrm{H}_{2}$ diminui os efeitos do aumento de pressão do sistema causado pelo acréscimo do número de mols durante a reação. Logo, membranas revestidas por paládio estão em uso na produção de hidrogênio e têm significativa importância na economia da indústria química usando PSM na produção de hidrogênio ultrapuro [18]. A partir deste contexto, torna-se atraente o desenvolvimento de membranas contendo Pd, analisando suas propriedades em processos de separação de gases. Neste trabalho são analisados os parâmetros de permeabilidade, seletividade, morfologia e composição química do suporte cerâmico e das membranas de compósitos $\mathrm{Pd} / \alpha-\mathrm{Al}_{2} \mathrm{O}_{3}$.

\section{MATERIAIS E MÉTODOS}

Materiais: os suportes de $\alpha$-alumina utilizados com dimensões de $104 \mathrm{~mm}$ de comprimento, 10 e $7 \mathrm{~mm}$ de diâmetro externo e interno, respectivamente, e tamanho de poros de cerca de $0,0055 \mu \mathrm{m}$ foram sinterizados a $1600{ }^{\circ} \mathrm{C}$ e fornecidos pela Tecnicer Celebra (Brasil). Para a limpeza dos suportes foram utilizadas soluções de $\mathrm{NaOH} 0,1 \%$ $(\mathrm{m} / \mathrm{v})$ e álcool isopropílico 99,5\% (Vetec). A solução solgel foi preparada com nitrato de alumínio nono-hidratado $\left[\mathrm{Al}\left(\mathrm{NO}_{3}\right)_{3} \cdot 9 \mathrm{H}_{2} \mathrm{O}\right] 98 \%$, ácido nítrico $\left(\mathrm{HNO}_{3}\right) 65 \%$ (Merck) 
e álcool polivinílico (PVA) 89,5\% (Vetec). A solução de sensibilização foi preparada utilizando cloreto de estanho II (98\%, Cinética), com ácido clorídrico ( $\mathrm{HCl}$ ) 37-38\% . Para a solução de ativação, utilizou-se cloreto de paládio II ( $\left.\mathrm{PdCl}_{2}\right)$ $60 \%$ com ácido clorídrico $(\mathrm{HCl}) 37-38 \%$. A solução para o electroless plating foi preparada com Titriplex II (EDTA, Merck, grau analítico), hidróxido de amônio $\left(\mathrm{NH}_{4} \mathrm{OH}\right) 24-$ $26 \%$, hipofosfito de sódio hidratado $\left(\mathrm{NaH}_{2} \mathrm{PO}_{2} \cdot \mathrm{H}_{2} \mathrm{O}\right) 98 \%$ e cloreto de paládio II $\left(\mathrm{PdCl}_{2}\right) 60 \%$. Os gases utilizados para os ensaios de permeação foram dióxido de carbono, metano e hidrogênio $\left(\mathrm{CO}_{2}, \mathrm{CH}_{4}\right.$ e $\left.\mathrm{H}_{2}\right)$ fornecidos pela Air Products com $99,9 \%$ de pureza.

Preparação das membranas: para remoção de possíveis impurezas e contaminantes dos suportes cerâmicos foi realizada limpeza conforme descrito em $[19,20]$. As extremidades do suporte foram fechadas com tampões de silicone e a solução de PVA 4\% depositada externamente ao suporte, pois foi na superfície externa do tubo cerâmico que foram depositadas as camadas de paládio. Em seguida, os suportes foram secos a $120{ }^{\circ} \mathrm{C}$ por $2 \mathrm{~h}$. Estes foram submetidos a uma sequência de etapas de imersão em soluções de sensibilização, ativação e deposição metálica. As etapas de sensibilização e ativação foram repetidas de forma intercaladas por dez vezes, sempre com as extremidades dos suportes fechadas. Os suportes foram imersos em solução de sensibilização por 2 min e lavados com água deionizada por $30 \mathrm{~s}$. Após as etapas de sensibilização e ativação, os suportes foram imersos na solução electroless plating. O processo de revestimento foi realizado a $50{ }^{\circ} \mathrm{C}$ durante $90 \mathrm{~min}$ e $\mathrm{pH} 11$. Os suportes cerâmicos com paládio foram mantidos em mufla a $600{ }^{\circ} \mathrm{C}$ por $3 \mathrm{~h}$. O processo electroless plating foi repetido duas vezes, correspondente a uma (MPd1) e duas camadas de paládio (MPd2).

Caracterização do suporte cerâmico e das membranas: o suporte cerâmico (MPd0) e as membranas (MPd1 e MPd2) foram caracterizados por microscopia eletrônica de varredura (MEV) e a caracterização química foi realizada por espectroscopia por dispersão de energia (EDS) com microscópio Shimadzu, SSX-550 Super-Scan. Amostra em pó do tubo cerâmico foi analisada no difratômetro de raios $\mathrm{X}$ da Shimadzu, XRD-6000, com radiação $\mathrm{CuK} \alpha, \lambda=1,5406$ Å. O tamanho médio e a distribuição de poros foram determinados pela análise de porosimetria por intrusão de mercúrio, realizada em porosímetro Quantachrome, PM60-17. Os ensaios de permeação com os gases dióxido de carbono, metano e hidrogênio $\left(\mathrm{CO}_{2}, \mathrm{CH}_{4}\right.$ e $\left.\mathrm{H}_{2}\right)$ foram realizados individualmente, em uma faixa de 1 a 4 bar de pressão. A taxa de saída do gás concentrado foi fixada em 1 L.min ${ }^{-1}$. O volume de gás permeado foi medido com gasômetro (LAO, G0,6) a $\sim 23{ }^{\circ} \mathrm{C}$, com variação da pressão de alimentação de 1 a 4 bar através da membrana (Fig. 1). Para as amostras com deposição de Pd foi utilizado o software ImageJ, com o qual foi possível medir aproximadamente a espessura da camada de Pd. A permeabilidade de hidrogênio foi calculada a partir da Eq. C e para o cálculo de seletividade das membranas foi utilizada a Eq. F:

$$
\alpha_{\mathrm{A} / \mathrm{B}}=\frac{\mathrm{P}_{\mathrm{A}}}{\mathrm{P}_{\mathrm{B}}}
$$

sendo $\mathrm{P}_{\mathrm{A}}$ a permeabilidade do gás de interesse $\left(\mathrm{H}_{2}\right)$ e $\mathrm{P}_{\mathrm{B}}$ a permeabilidade dos demais gases $\left(\mathrm{CO}_{2}\right.$ e $\left.\mathrm{CH}_{4}\right)$.

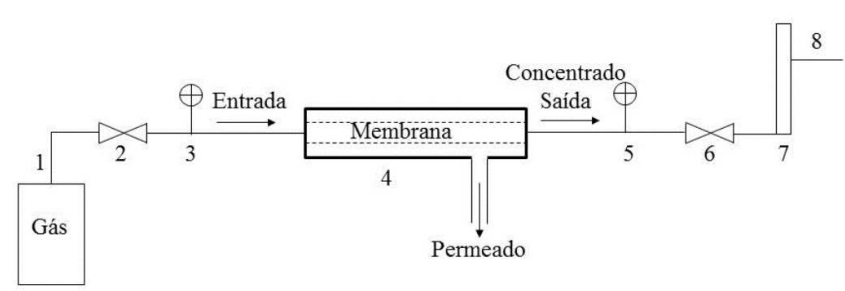

Figura 1: Fluxograma do sistema de permeação de gases: 1cilindro de gás; 2 e 6- válvula reguladora; 3 e 5- manômetro; 4célula de aço inox com membrana tubular; 7- rotâmetro; e 8- saída do concentrado.

[Figure 1: Schematic diagram of gas permeation system: 1- gas cylinder; 2 and 6-regulating valve; 3 and 5-pressure gauge; 4stainless steel cell with tubular membrane; 7-rotameter; and 8concentrate output.]

\section{RESULTADOS E DISCUSSÃO}

A caracterização do suporte cerâmico (MPd0) realizada por EDS mostrou que a composição química do suporte cerâmico de $\alpha$-alumina $\left(\mathrm{Al}_{2} \mathrm{O}_{3}\right)$ contém ainda ferro $\left(\mathrm{Fe}_{2} \mathrm{O}_{3}\right)$ e silício $\left(\mathrm{SiO}_{2}\right.$, Fig. 2a). Os demais elementos como carbono, cálcio, magnésio e sódio podem ocorrer como contaminantes [21]. A presença de ouro é devido à deposição desse metal sobre a amostra para realização da análise. A Fig. 2b mostra em cor amarela os sinais emitidos pelo alumínio do $\mathrm{Al}_{2} \mathrm{O}_{3}$. A Fig. 2c ilustra o difratograma de raios X do suporte cerâmico (MPd0) sinterizado a 1600 ${ }^{\circ} \mathrm{C}$, no qual se observa a presença de um sistema cristalino hexagonal $\alpha$-alumina com picos característicos de $2 \theta$ em $25,35,37,43,53,57,66,68$ e $77^{\circ}$, de acordo com o International Centre of Diffraction Data (2003) por meio do arquivo cristalográfico ICSD n ${ }^{\circ} 10425$.

A distribuição de tamanho de poro para o suporte cerâmico em estudo pode ser visualizada na Fig. 3a, a qual apresenta a plotagem da derivada da curva cumulativa, $-\mathrm{dV} / \mathrm{d}(\log \mathrm{d})$, versus diâmetro de poros. $\mathrm{O}$ gráfico mostra que o diâmetro de poro com maior frequência é $0,0055 \mu \mathrm{m}$. A curva de distribuição do volume de $\mathrm{Hg}$ intrudado em função do diâmetro de poros está apresentada na Fig. 3b, onde o volume máximo de $\mathrm{Hg}$ intrudado $\left(0,0081 \mathrm{~cm}^{3} \cdot \mathrm{g}^{-1}\right)$ correspondeu aos poros com menores diâmetros. Durante a sinterização $\left(1600{ }^{\circ} \mathrm{C}\right)$ ocorreu a redução da porosidade e a densificação, com o crescimento dos grãos [22], justificando o diâmetro de poro encontrado $(\sim 5 \mathrm{~nm})$ no suporte cerâmico e volume máximo de mercúrio $(\mathrm{Hg})$ intrudado de $0,0081 \mathrm{~cm}^{3} \cdot \mathrm{g}^{-1}$.

As análises realizadas por MEV mostram os grãos na superfície do suporte cerâmico sem nenhum recobrimento na Fig. 4a, enquanto que na Fig. 4b a superfície está recoberta pela solução sol-gel, a qual conferiu uma camada 

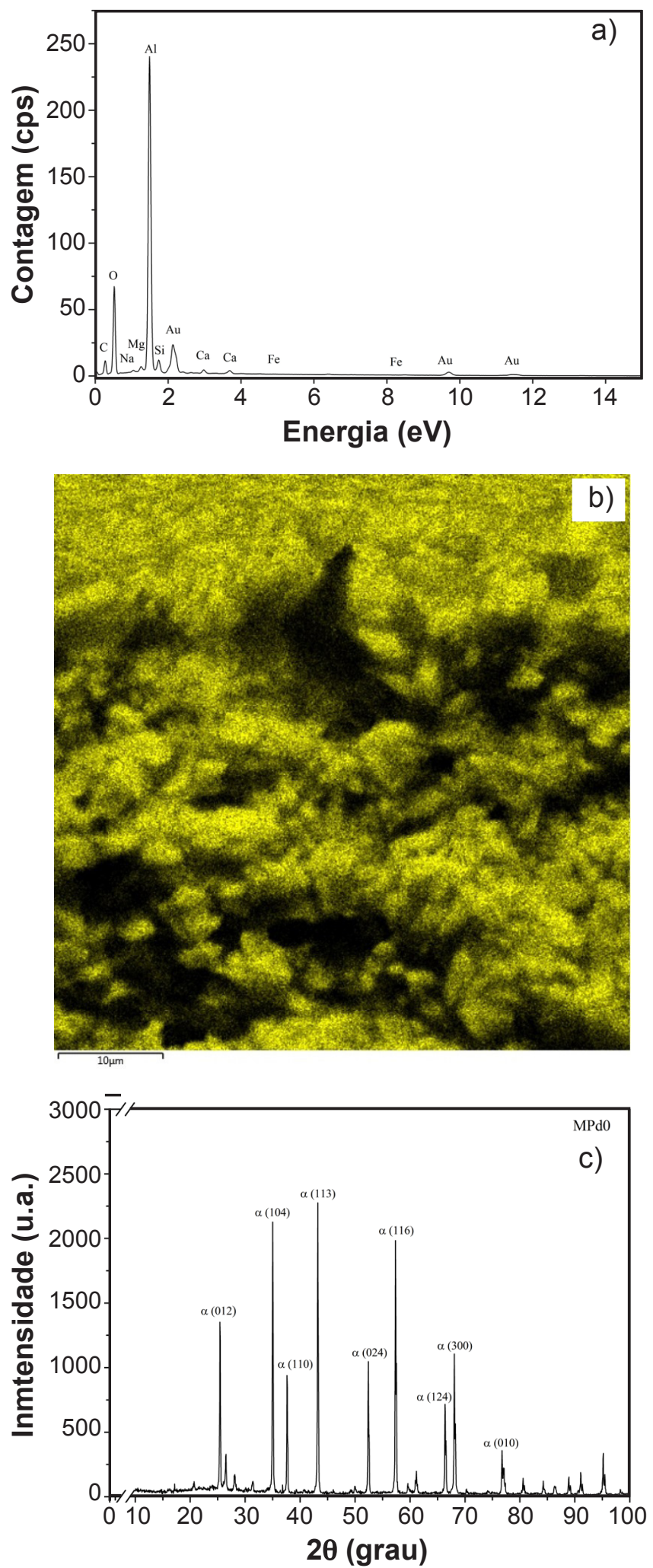

Figura 2: Análise da composição química do suporte cerâmico (MPd0) sem a deposição de paládio realizada por EDS (a), mapeamento do alumínio (Al) por EDS (b) e difratograma de raios $\mathrm{X}$ mostrando presença de fase $\alpha$-alumina (c).

[Figure 2: Chemical composition analysis of ceramic support without palladium deposition (MPdO) by EDS (a), mapping of aluminum (Al) by EDS (b), and X-ray diffraction pattern showing a-alumina phase (c).] bem distribuída sobre o suporte cerâmico, que segundo [23] afeta diretamente a uniformidade da camada de paládio a ser depositada. Quanto mais uniforme e menos imperfeições existirem na camada superior do suporte, mais regular
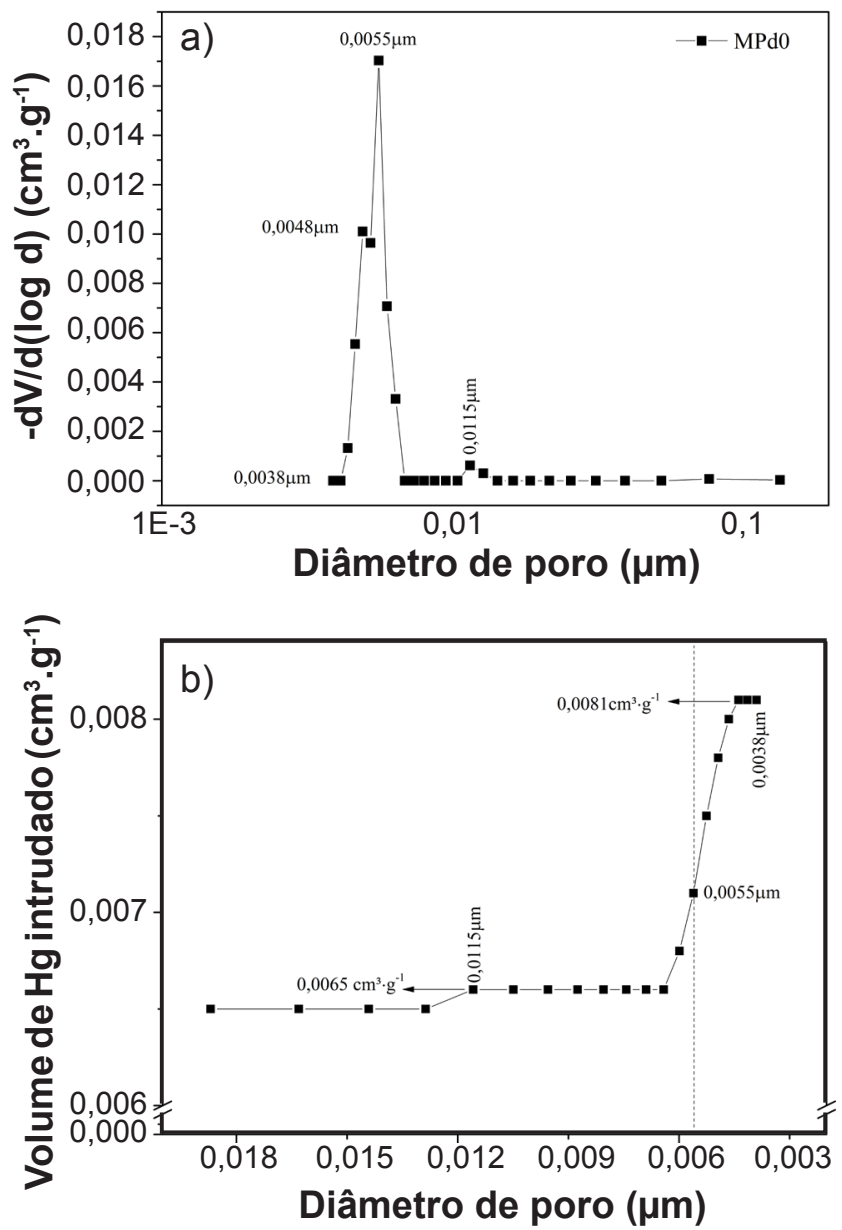

Figura 3: Curva de distribuição do diâmetro de poros obtido por porosimetria por intrusão de mercúrio (a) e curva de distribuição do volume de $\mathrm{Hg}$ intrudado versus diâmetro de poros (b) para o suporte cerâmico (MPd0) de $\alpha$-alumina.

[Figure 3: Pore size distribution curve obtained by mercury intrusion porosimetry (a) and distribution curve of the Hg volume intruded versus pore diameter (b) for the a-alumina ceramic support (MPd0).]
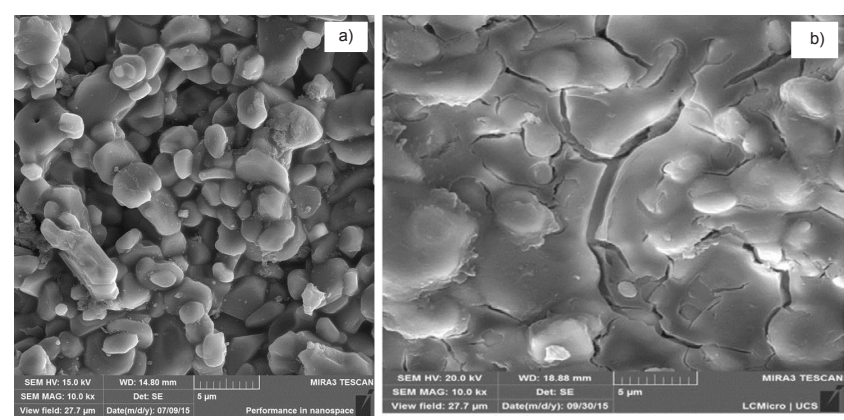

Figura 4: Micrografias (MEV) da superfície do suporte cerâmico puro (a) e do suporte cerâmico submetido ao método sol-gel (b). [Figure 4: Micrographs (SEM) of pure ceramic support surface (a) and the ceramic support submitted to the sol-gel method (b).] 
é a deposição de paládio, aperfeiçoando a eficiência da membrana. Na Fig. 4b são observadas pequenas trincas e caminhos na camada sol-gel que podem facilitar o transporte dos gases através do suporte devido ao seu diâmetro cinético.

A análise da composição química da membrana com uma camada de Pd (MPd1) pode ser observada na Fig. 5a, a qual mostra que o recobrimento do suporte cerâmico com Pd por electroless plating reduziu a intensidade do sinal dos elementos do suporte cerâmico, principalmente alumínio e oxigênio, porém não interferiu na intensidade do sinal do carbono. Possivelmente isso tenha ocorrido devido à presença de C na solução de PVA. A Fig. 5 b mostra a presença de carbono (vermelho) na membrana com uma deposição de Pd. A espessura da camada de paládio foi determinada pela análise de EDS da membrana com uma camada (MPd1) ou duas camadas de paládio (MPd2). Pela imagem da Fig. 6a obteve-se uma camada de Pd de aproximadamente 0,8 $\mu \mathrm{m}$ (azul). Para a membrana com 2 camadas de Pd, Fig. $6 \mathrm{~b}$, a espessura foi de aproximadamente $1,3 \mu \mathrm{m}$ (azul). A espessura da camada de Pd variou ao longo da seção devido
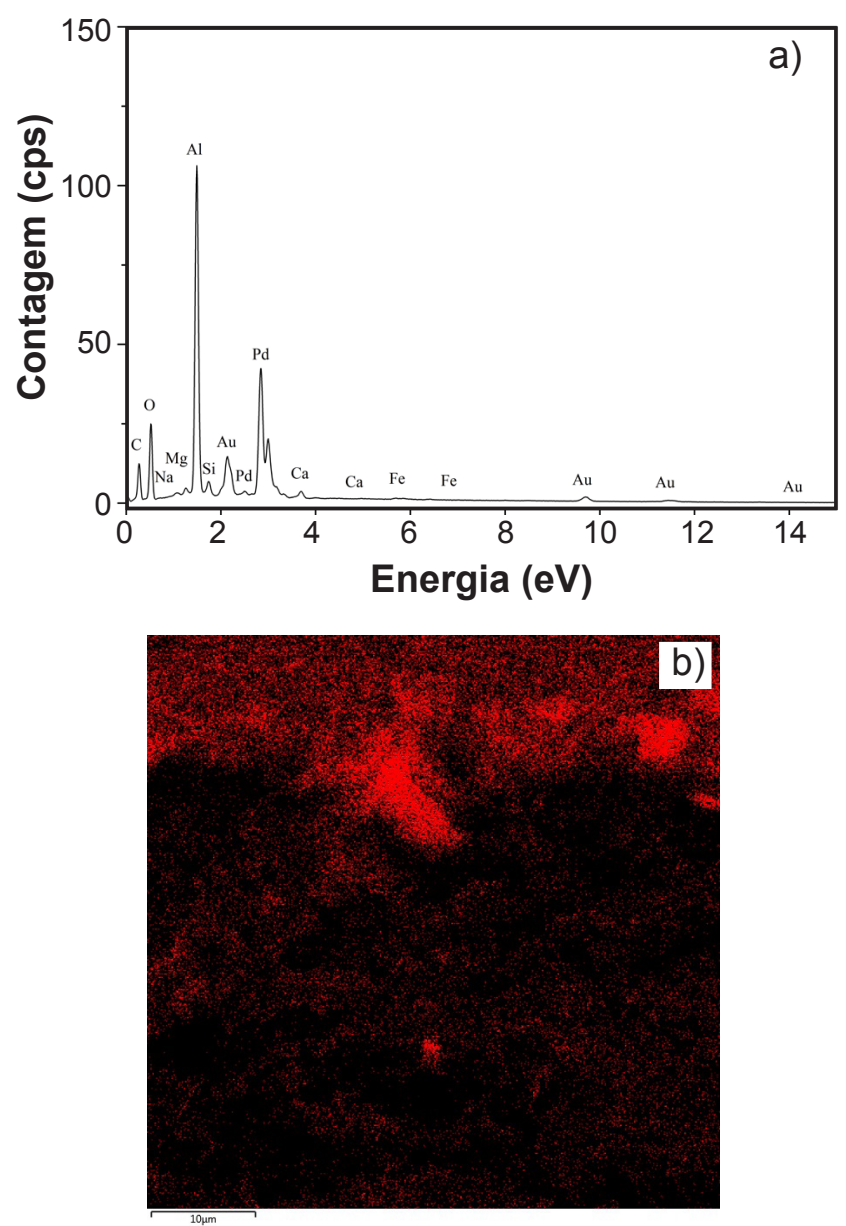

Figura 5: Análise da composição química da membrana com uma camada de paládio (MPd1) (a) e mapeamento por EDS da membrana alumina/Pd com uma camada mostrando a presença de C (vermelho) (b).

[Figure 5: Membrane chemical composition analysis with one palladium layer (MPd1) (a) and EDS mapping of alumina/Pd membrane with one layer showing the presence of $C(\mathrm{red})(b)$.

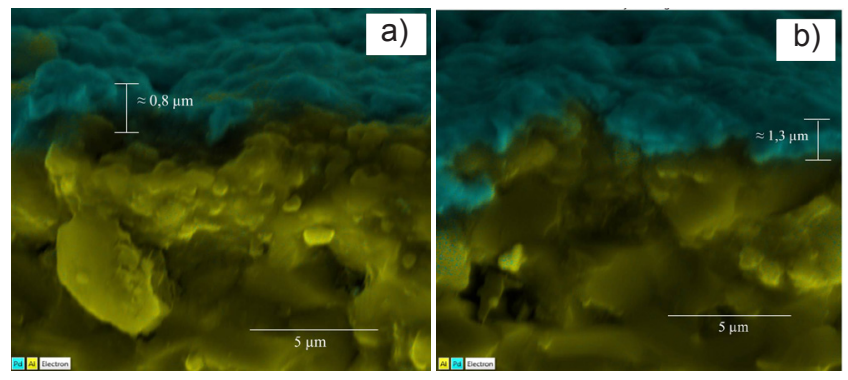

Figura 6: Micrografias (MEV) da secção transversal de membrana com: (a) uma camada de Pd (MPd1); e (b) duas camadas de Pd (MPd2).

[Figure 6: Micrographs (SEM) of the cross section of the membrane with: (a) one Pd layer (MPd1); and (b) two Pd layers (MPd2).]

à diferença entre o tamanho do grão da alumina e do $\mathrm{Pd}$ tornando a superfície do tubo cerâmico irregular (em grande aumento) e consequentemente da camada de Pd.

A Fig. 7a mostra a superfície da membrana MPd1, na qual se observa uma deposição uniforme ao longo da membrana com a presença de algumas microtrincas na sua estrutura. $\mathrm{Na}$ Fig. 7b é apresentada a superfície da membrana MPd2. Esta membrana não apresentou uniformidade em sua superfície, sendo suscetível a trincas (falhas) que podem comprometer a eficiência da membrana, como pode ser observado na Fig. $7 \mathrm{~d}$ com maior magnificação. A imagem por MEV do suporte cerâmico com uma camada de Pd, Fig. 7c, mostrou a presença de alguns vazios. Após ocorrer a deposição da segunda camada de Pd, Fig. 7d, aumentou a quantidade e o tamanho dos vazios, reduzindo a uniformidade da estrutura. Segundo [24], a cada deposição de Pd, aumenta-se o número de vazios, que são recobertos por uma nova camada, e assim sucessivamente.

As membranas foram caracterizadas quanto à
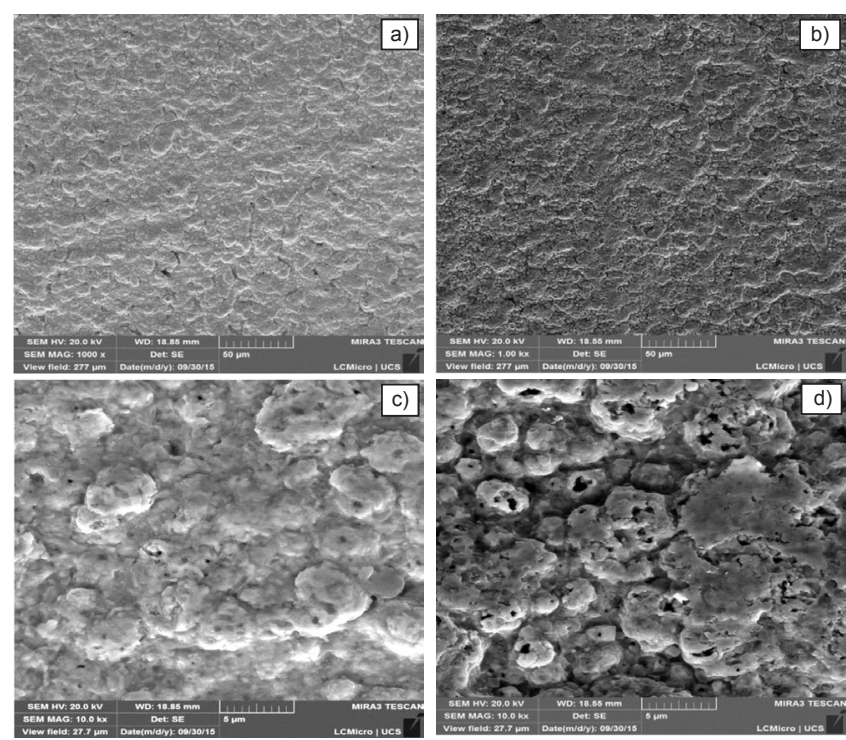

Figura 7: Micrografias (MEV) da superfície da membrana cerâmica com: (a,c) uma camada de Pd (MPd1); e (b,d) duas camadas de Pd (MPd2).

[Figure 7: Micrographs (SEM) of the ceramic membrane surface with: (a,c) one Pd layer (MPd1); and (b) two Pd layers (MPd2).] 
permeabilidade dos gases considerando o fluxo permeado. O suporte cerâmico sem deposição de $\mathrm{Pd}$ (MPd0) bem como as membranas preparadas para os ensaios foram submetidos à permeação do gás de interesse $\left(\mathrm{H}_{2}\right)$ durante 15 min para garantir o equilíbrio entre o gás e a estrutura da membrana. A Fig. 8 apresenta os resultados de permeabilidade por meio da variação da pressão de alimentação para os gases
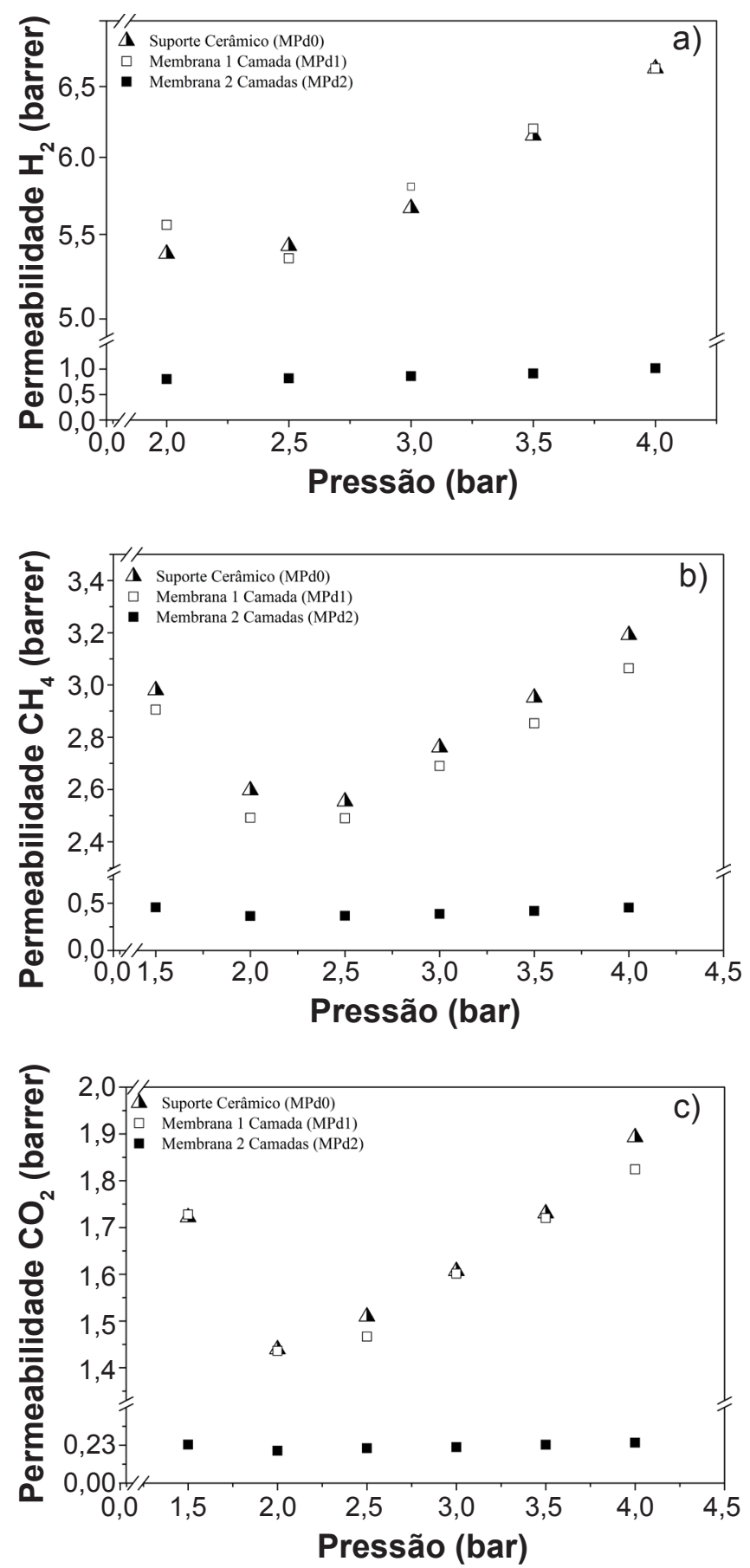

Figura 8: Permeabilidade dos gases $\mathrm{H}_{2}$ (a), $\mathrm{CH}_{4}$ (b) e $\mathrm{CO}_{2}$ (c) em função da pressão de alimentação, nas membranas cerâmicas com e sem Pd.

[Figure 8: Permeability of gas $\mathrm{H}_{2}\left(\right.$ a) $\mathrm{CH}_{4}\left(\right.$ b) and $\mathrm{CO}_{2}$ (c) as a function of the feed pressure in ceramic membranes with and without Pd.]
$\mathrm{H}_{2}, \mathrm{CH}_{4}$ e $\mathrm{CO}_{2}$. A permeabilidade dos gases foi calculada a partir da Eq. C. Os ensaios de permeação mostraram a seletividade que a camada de paládio confere à membrana para o gás hidrogênio, visto que para todas as condições ensaiadas houve maior permeação ao gás hidrogênio em relação aos demais gases estudados. Pode ser observado que com o aumento da pressão de entrada do gás no sistema de permeação aumentou a permeabilidade do gás ensaiado, porém a permeabilidade diminuiu com o aumento no número de camadas depositadas de Pd. A permeabilidade da membrana MPd2 reduziu aproximadamente 15\% para os três gases testados e apresentou comportamento linear como aumento da pressão. Os valores de permeabilidade podem estar relacionados à diferença do diâmetro cinético entre as moléculas dos gases testados. Moléculas menores difundem mais facilmente na estrutura cristalina do Pd e também na estrutura porosa do suporte, o que é justificado pelo diâmetro cinético das moléculas de $0,29 \mathrm{~nm}$ para $\mathrm{H}_{2}$, $0,33 \mathrm{~nm}$ para $\mathrm{CO}_{2}$ e $0,38 \mathrm{~nm}$ para $\mathrm{CH}_{4}$ [25]. O aumento do fluxo permeado de $\mathrm{H}_{2}$ em relação aos demais gases analisados está também relacionado à afinidade que o $\mathrm{Pd}$ possui com o $\mathrm{H}_{2}$. Segundo [5], o hidrogênio é dissociado pelo Pd e, quando em sua forma metálica, absorve $\mathrm{H}_{2}$ gasoso em quantidade de até 600 vezes o seu volume mantendo suas propriedades físicas e sua integridade estrutural.

De acordo com [26], o desempenho da membrana é proporcional à espessura da camada de Pd: quanto mais fina a espessura da camada, maior é o fluxo de gás obtido. Autores [26] relataram que grãos menores formam filmes mais densos e melhoram a seletividade, ao contrário de grãos maiores que diminuem a eficiência do transporte seletivo, que é o caso da membrana MPd1 (Fig. 8a), que apresentou permeabilidade praticamente idêntica ao suporte cerâmico sem deposição de Pd (MPd0). Athayde et al. [27] utilizaram filmes de $\mathrm{Pd} / \mathrm{Ag}$ suportados em poli(dimethilsiloxane) (PDMS) e obtiveram resultados semelhantes aos encontrados neste trabalho. Os fluxos permeados do suporte e da membrana com uma camada ficaram muito próximos e com duas camadas diminuiu tanto para o gás $\mathrm{H}_{2}$ quanto para o $\mathrm{CO}_{2}$. Este trabalho se diferencia do trabalho [27] por utilizar como suporte um tubo de $\alpha$-alumina, que em função de sua temperatura de sinterização $\left(1600{ }^{\circ} \mathrm{C}\right)$ pode funcionar como barreira seletiva e com apenas duas camadas de Pd apresentou resultados interessantes a baixas pressões e temperatura, além de ser mais resistente a deformações que o suporte polimérico apresentado. Os valores obtidos para os coeficientes angulares da reta a partir dos resultados de permeabilidade (Fig. 8) estão apresentados na Tabela I.

A seletividade aparente, ou intrínseca, foi calculada a partir dos testes realizados, porém a real seletividade apenas pode ser comprovada por meio de testes com mistura de gases de composições conhecidas. A Tabela II apresenta os valores de seletividade aparente $(\alpha)$ obtidos para cada amostra, de acordo com o gás de interesse (hidrogênio) e comparado com os demais gases. Os ensaios mostraram que a permeabilidade do suporte cerâmico 
Tabela I - Coeficiente angular da reta de permeabilidade para os gases $\mathrm{H}_{2}, \mathrm{CH}_{4}$ e $\mathrm{CO}_{2}$.

[Table I - Slope coefficient for $\mathrm{H}_{2}, \mathrm{CH}_{4}$ and $\mathrm{CO}_{2}$ gas permeability.]

\begin{tabular}{cccc}
\hline Amostra & $\begin{array}{c}\mathrm{H}_{2} \\
\text { (barrer) }\end{array}$ & $\begin{array}{c}\mathrm{CH}_{4} \\
\text { (barrer) }\end{array}$ & $\begin{array}{c}\mathrm{CO}_{2} \\
\text { (barrer) }\end{array}$ \\
\hline MPd0 & 0,320 & 0,133 & 0,104 \\
MPd1 & 0,318 & 0,118 & 0,084 \\
MPd2 & 0,033 & 0,009 & 0,010 \\
\hline
\end{tabular}

sem deposição de Pd (MPd0) foi semelhante à membrana com uma camada de Pd (MPd1) e superior à membrana com duas camadas de Pd (MPd2). Isso comprovou que a sinterização da $\alpha$-alumina realizada a $1600^{\circ} \mathrm{C}$ é uma ótima condição para os tubos serem utilizados como suporte que, de acordo com [23], quanto menor o tamanho de poro do suporte, menor é o custo na formação da camada seletiva, pois reduz em muito o número de camadas de Pd e mais eficiente é o processo. Bottino et al. [28] observaram que a permeação de gás diminui com o aumento da espessura da camada de paládio, e que a espessura necessária para atingir valores significativos de permeação seria com camadas mais finas, justificando a redução na permeação da membrana MPd2 encontrada nesse trabalho. As membranas preparadas (Tabela II) apresentaram resultados de seletividade $(\alpha)$ em relação ao $\mathrm{H}_{2} \geq 1$. Para o par $\mathrm{H}_{2}$ / $\mathrm{CH}_{4}$ a seletividade aumentou com o aumento do número de camadas de Pd. Para a membrana MPd1 $(\alpha=2,68)$ foi $\sim 12 \%$ maior em relação ao MPd0 $(\alpha=2,40)$ e para MPd2 $(\alpha=3,43)$ foi $\sim 28 \%$ maior em relação a MPd1 $(\alpha=2,68)$ para o gás $\mathrm{H}_{2}$. Para o par $\mathrm{H}_{2} / \mathrm{CO}_{2}$ a seletividade foi $\sim 23 \%$ maior para a membrana MPd1 $(\alpha=3,79)$ em relação a MPd0 $(\alpha=3,07)$ e $\sim 25 \%$ em relação a MPd2 $(\alpha=3,02)$ para o gás $\mathrm{H}_{2}$. Esse resultado pode ser justificado pela estrutura das camadas de Pd (Figs. 7c e 7d) e pelo diâmetro cinético das moléculas $\left(0,33 \mathrm{~nm}\right.$ para $\mathrm{CO}_{2}$ e $0,38 \mathrm{~nm}$ para $\left.\mathrm{CH}_{4}\right)$. Athayde et al. [27] trabalharam com membranas de $\mathrm{Pd} / \mathrm{Ag} /$ PDMS e encontraram seletividade $\alpha=1$ para $\mathrm{H}_{2} / \mathrm{CO}_{2}$ para o suporte e $\alpha=10$ para $\mathrm{H}_{2} / \mathrm{CO}_{2}$ para a membrana com duas camadas, mostrando que o tipo de suporte influencia na seletividade da membrana.

Tabela II - Resultados de seletividade $(\alpha)$ em relação ao gás $\mathrm{H}_{2}$. [Table II - Selectivity $(\alpha)$ results in relation to $\mathrm{H}_{2}$ gas.]

\begin{tabular}{ccc}
\hline Amostra & $\mathrm{H}_{2} / \mathrm{CH}_{4}$ & $\mathrm{H}_{2} / \mathrm{CO}_{2}$ \\
\hline MPd0 & 2,40 & 3,07 \\
MPd1 & 2,68 & 3,79 \\
MPd2 & 3,43 & 3,02 \\
\hline
\end{tabular}

\section{CONCLUSÕES}

De acordo com as análises realizadas, as membranas preparadas com tubos de $\alpha$-alumina revestidos com camadas de Pd por electroless plating apresentaram permeabilidade a $\mathrm{H}_{2}>\mathrm{CH}_{4}>\mathrm{CO}_{2}$ e grau de seletividade $\alpha$ de 3,07 para $\mathrm{H}_{2} / \mathrm{CO}_{2}$ para o suporte e $\alpha=3,79$ para a membrana com uma camada de Pd. Para $\mathrm{H}_{2} / \mathrm{CH}_{4}$, obteve-se $\alpha=2,40$ para o suporte e $\alpha=3,43$ para a membrana com duas camadas. Estes resultados podem ser considerados satisfatórios, visto que os ensaios foram realizados a $\sim 23{ }^{\circ} \mathrm{C}$ com pressões de 1 a 4 bar.

\section{AGRADECIMENTOS}

Os autores agradecem à Universidade de Caxias do Sul/ PGEPROTEC e ao CNPq pelo apoio e bolsas concedidas.

\section{REFERÊNCIAS}

[1] D. VanHolt, E. Forster, M.E. Ivanova, W.A. Meulenberg, M. Müller, S. Baumann, R. Vaßen, J. Eur. Ceram. Soc. 34 (2014) 2381.

[2] M. Vadrucci, F. Borgognoni, A. Moriani, A. Santucci, S. Tosti, Int. J. Hydrogen Energy 38 (2013) 4144.

[3] P. Pandey, R.S. Chauhan, Prog. Polym. Sci. 26 (2001) 853. [4] J.P. Collins, J.D. Way, Ind. Eng. Chem. Res. 32 (1993) 3006.

[5] N.A. Al-Mufachi, N.V. Rees, R. Steinberger-Wilkens, Sustain. Energy Rev. 47 (2015) 540.

[6] T.L. Ward, T. Dao, J. Memb. Sci. 153 (1999) 211.

[7] I.J. Iwuchukwu, A. Sheth, Chem. Eng. Process. Process. Intensif. 47 (2008) 1298.

[8] F.C. Gielens, H.D. Tong, M.A.G. Vorstman, J.T.F. Keurentjes, J. Memb. Sci. 289 (2007) 15.

[9] C.J. Bernardes, "Estudo da permeação de hidrogênio em reator com membrana de paládio: modelagem matemática e simulação computacional”, Diss. Mestr., Un. Fed. Uberlândia (2009).

[10] N. Vicinanza, I.-H. Svenum, L.N. Naess, T.A. Peters, R. Bredesen, A. Borg, H.J. Venvik, J. Memb. Sci. 476 (2015) 602.

[11] M. Mulder, Basic principles of membrane technology, $2^{\text {nd }}$ ed., Kluwer Acad. Publ., London (2003).

[12] A.G. Gil, M.H.M. Reis, D. Chadwick, Z. Wu, K. Li, Int. J. Hydrogen Energy 40 (2015) 3249.

[13] D.A.P. Tanaka, M.A.L. Tanco, S. Niwa, Y. Wakui, F. Mizukami, T. Namba, T.M. Suzuki, J. Memb. Sci. 247 (2005) 21.

[14] S. Yun, J.H. Ko, S.T. Oyama, J. Memb. Sci. 369 (2011) 482.

[15] B.D. Morreale, M.V. Ciocco, R.M. Enick, B.I. Morsi, B.H. Howard, A.V. Cugini, K.S. Rothenberger, J. Memb. Sci. 212 (2003) 87.

[16] V. Höllein, M. Thornton, P. Quicker, R. Dittmeyer, Catal. Today 67 (2001) 33.

[17] A. Basile, L. Paturzo, F. Laganà, Catal. Today 67 (2001) 65.

[18] J. Boon, J.A.Z. Pieterse, F.P.F. van Berkel, Y.C. van Delft, M. van Sint Annaland, J. Memb. Sci. 496 (2015) 344.

[19] R. Sari, Z. Yaakob, M. Ismail, W.R.W. Daud, J. Appl. Sci. 10 (2010) 1151. 
[20] R. Sari, Z. Yaakob, M. Ismail, W.R.W. Daud, L. Hakim, Ceram. Int. 39 (2013) 3211.

[21] P. Patnaik, Handbook of inorganic chemicals, McGrawHill (2003).

[22] D.W. Richerson, Modern ceramic engineering: properties, processing, and use in design, $2^{\text {nd }}$ ed., Marcel Dekker, New York (1992).

[23] T. Bisoto, "Preparação e caracterização de membranas cerâmicas compostas tubulares para aplicação na separação de gases", Diss. Mestr., Un. Caxias Sul (2014).

[24] J.P. Wu, I.W.M. Brown, M.E. Bowden, T. Kemmitt, Solid State Sci. 12 (2010) 1912.
[25] J.S. Nascimento, "Separação de hidrogênio de misturas gasosas através de membranas de carbono do tipo fibra oca", Diss. Mestr., Un. Fed. Rio Janeiro (2010).

[26] E. David, J. Kopac, Int. J. Hydrogen Energy 36 (2011) 4498.

[27] A.L. Athayde, R.W. Baker, P. Nguyen, J. Membr. Sci. 94 (1994) 299.

[28] A. Bottino, M. Broglia, G. Capannelli, A. Comite, P. Pinacci, M. Scrignari, F. Azzurri, Int. J. Hydrogen Energy 39 (2014) 4717.

(Rec. 14/11/2016, Rev. 25/05/2017, 08/12/2017, Ac. 21/03/2018) 